but both trials were stopped early because of a lack of benefit or evidence of harm in the active treatment groups.

During the first year of therapy, the incidence of urinary incontinence was significantly higher among women receiving either type of menopausal hormone therapy than in the placebo groups. This was the case for stress incontinence and mixed incontinence, although the incidence of urge incontinence was not affected by estrogen plus progestin therapy. Participants who had urinary incontinence at baseline were at increased risk of worsening amount and frequency of urinary incontinence, compared with those on placebo. In addition, the symptoms of urinary incontinence limited daily activities and bothered or disturbed these women more frequently than reported in the placebo group.

Based on these results, Hendrix et al. advise against using estrogen or estrogen plus progestin in the prevention or treatment of urinary incontinence.

Original article Hendrix SL et al. (2005) Effects of estrogen with and without progestin on urinary incontinence. JAMA 293: 935-948

\section{Is amoxicillin-clavulanate as effective as ciprofloxacin in female acute cystitis?}

Current standard therapy for acute uncomplicated cystitis in women comprises trimethoprim-sulfamethoxazole for 3 days. As the prevalence of resistance to this combination grows in some areas, increasing reliance on fluoroquinolones as an alternative strategy for this and other infections, such as respiratory tract infections, is raising concerns about the future usefulness of this drug class. Although increasing emphasis is being placed on the use of fluoroquinolone-sparing regimes, no published studies have evaluated the efficacy of amoxicillin-clavulanate in the current climate of high-level amoxicillin resistance.

Hooton et al. compared the efficacy of 3-day regimens of amoxicillin-clavulanate and ciprofloxacin in a randomized, single-blind trial in 370 women aged 18-45 years with symptoms of acute uncomplicated cystitis. Patients were recruited between July 1998 and May 2002 from a university student health center and a health maintenance organization and were followed up for 4 months.

The rate of clinical cure (defined as lack of symptomatic persistent or recurrent urinary tract infection) was significantly lower in the group receiving amoxicillin-clavulanate (58\%) compared with the group given ciprofloxacin (77\%). The difference remained significant even when women with strains of Escherichia coli susceptible to amoxicillin-clavulanate were analyzed separately. The difference in clinical cure rates was most marked during the first 2 weeks of therapy.

The authors conclude that trimethoprimsulfamethoxazole should remain first-line treatment for acute cystitis in women with no history of allergy to the combination and where the risk of resistance is low. Use of a fluoroquinolone or other alternative, such as nitrofurantoin, is appropriate in situations where trimethoprim-sulfamethoxazole resistance is likely. Amoxicillin-clavulanate is not an appropriate first-line agent for empiric therapy of acute cystitis.

Original article Hooton TM et al. (2005) Amoxicillinclavulanate vs ciprofloxacin for the treatment of uncomplicated cystitis in women: a randomized trial. 949-955

\section{Sperm aneuploidy and male factor infertility}

Studies of patients with oligoasthenoteratozoospermia-or a low sperm count with low motility and a high proportion of abnormal sperm-have revealed a link between male infertility and the risk of chromosomal abnormalities in sperm nuclei. Recently, it has been suggested that high sperm aneuploidy might decrease the likelihood of successful intracytoplasmic sperm injection (ICSI). François Petit and colleagues asked, therefore, whether sperm aneuploidy determination is a useful predictive test in patients undergoing ICSI.

The team compared sperm aneuploidy rates in 10 men who had had at least four unsuccessful ICSI treatments (group A), 9 men whose partners had become pregnant after up to three ICSI attempts (group B) and 10 fertile men with normal sperm concentration, motility and morphology (group C). The analysis was performed using fluorescence in situ 